\title{
Competencias para la Responsabilidad Social Universitaria: una Comparativa de Perspectivas entre Universidades
}

\author{
Jesus Águila-León ${ }^{\mathrm{a}}$, Cristian Chiñas-Palacios ${ }^{\mathrm{b}}$, Carlos Vargas-Salgado ${ }^{\mathrm{c}}$, Francisco Martínez $^{\mathrm{d}}$ \\ a,bInstituto Universitario de Ingeniería Energética, Universitat Politècnica de València, València, España; \\ 'Departamento de Ingeniería eléctrica, Universitat Politècnica de València, València, España; ${ }^{a, b}$ Departamento de \\ Estudios del Agua y la Energía, Centro Universitario de Tonalá, Universidad de Guadalajara, Jalisco, México; \\ dExperiencia Estudiantil, Coordinación de Responsabilidad Social, Universidad del Valle de México Campus \\ Zapopan, Jalisco, México. \\ a jeagleo@upvnet.upv.es, ${ }^{\mathrm{b}}$ cricripa@upvnet.upv.es, ${ }^{\mathrm{c}}$ carvarsa@upvnet.upv.es, ${ }^{\mathrm{d}}$ francisco_martinezsa@my.uvm.edu.mx
}

\begin{abstract}
The world faces essential challenges, including climate change, resource depletion and inequality. University, as an agent of change, must take part in the University Social Responsibility (USR) since it trains future professionals. This study's purpose is to evaluate the generic competencies related to the USR of university students to obtain criteria that help the generation of strategies in the benefit of society and the environment. The methodology used is based on the application of a survey for students from three universities: two Mexican, one public and one private, and one Spanish public university. The survey questions are based on the self-perception and behaviour of the student regarding the USR. The data obtained are statistically compared. The results show that the students of the Mexican private university and the Spanish public university have a high sense of the USR compared to the Mexican public university. The managers and teachers of the Mexican public university should consider paying more considerable attention to the development of the generic competencies related to the USR since these are determinants for the training of responsible citizens and collaborators of sustainability and a healthy environment.
\end{abstract}

Keywords: university social responsibility, USR, competencies, generic competencies, environment, sustainability.

\section{Resumen}

El mundo enfrenta importantes retos, entre ellos: cambio climático, agotamiento de recursos, desigualdad. La universidad como agente de cambio debe tomar parte de la Responsabilidad Social Universitaria (RSU), puesto que en ella se forma a los futuros profesionistas. El propósito de este estudio es evaluar las competencias transversales relacionadas con la RSU de alumnos universitarios para obtener criterios que ayuden a una futura generación de estrategias en beneficio de la sociedad y el medio ambiente. La metodología empleada se basa en la aplicación de una encuesta a estudiantes de tres universidades: dos mexicanas, una pública y una privada, y una pública española; las preguntas se basan en la autopercepción y comportamiento del encuestado respecto a la RSU. Los datos obtenidos son comparados estadísticamente. Los resultados muestran que los alumnos de la universidad privada mexicana y de la universidad pública española tienen un alto sentido de la RSU en comparación con la universidad pública mexicana. Los directivos y docentes de la universidad pública mexicana deberían considerar prestar mayor atención al desarrollo de las competencias genéricas relacionadas con la RSU, puesto que estas son determinantes para la formación de ciudadanos responsables y colaboradores de la sostenibilidad y el medio ambiente sano. 
Palabra clave: responsabilidad social universitaria, RSU, competencias, competencias genéricas, medio ambiente, sostenibilidad.

\section{Introducción}

En las universidades se forma a los futuros profesionistas, es allí donde adquieren los conocimientos y habilidades que les permitirán ejercer su profesión en el campo laboral. La universidad en España tiene un largo recorrido a través de los siglos, con casi 800 años de historia desde que el Studium Generale de Salamanca obtuvo el título de Universidad a través de la bula papal de Alejandro IV y la real orden de Alfonso X el Sabio un 9 de noviembre del año 1252 convirtiéndose así en la primera institución educativa europea en portar el título de Universidad (Universidad de Salamanca, 2020), presenciando y participado de las profundas transformaciones de España, desde entonces hasta nuestros días.

Por su parte en América, la historia de la Universidad comenzó el año de 1551 tanto en la Ciudad de México como en Lima, Perú. La Real Universidad de México se instituyó el 21 de septiembre de 1551 mediante la Real cédula del Rey Carlos I de España y V del Sacro Imperio Romano Germánico donde puede leerse "Tenemos por bien y es nuestra merced y voluntad que en la dicha ciudad de México pueda haber y haya el dicho estudio e Universidad la cual tenga e goce todos los privilegios y franquezas e libertades y exenciones que tiene e goza el Estudio e Universidad de la dicha ciudad de Salamanca" (UNAM, 2020), con lo cual se respondió a la creciente necesidad del Nuevo Mundo para la formación en artes, teología y ciencias que aquella época demandaba para los habitantes de esas tierras, actuando instrumento pacificador para crear armonía y una nueva identidad luego de la colonización. Fueron notables los breves pero importantes esfuerzos por incluir en la Universidad a los pueblos nativos, tanto en México como en el Perú, ofertándose clases en lenguas nativas (Leal \& Mira, 2016) para la nobleza indígena, con acceso a la universidad, y para los misioneros católicos que habrían de usar lenguas indígenas para la evangelización. De esta manera en México como en España, la Universidad ha sido testigo y actor de profundos cambios sociales a lo largo de centurias.

Es en esta praxis de las universidades de formar ciudadanos, actualizarse y actuar para los retos presentes de la sociedad en donde entra la Responsabilidad Social Universitaria (RSU) en la actualidad (Vallaeys, 2016). La RSU es el modo en que la universidad responde a los retos de nuestra sociedad, destacándose: el cambio climático, el agotamiento y contaminación de los recursos, las epidemias y la igualdad de género y de minorías. Las universidades tienen cuatro grandes impactos: organizacionales, educativos, cognitivos y sociales (Vallaeys, de la Cruz, \& Sasia, 2009). Los impactos organizacionales están referidos al campo laboral y ambiental; los impactos educativos con la formación académica; los impactos cognitivos con la investigación y epistemología y los impactos sociales con actividades de extensión, voluntariados, transferencia y proyección social. En la modernidad la Responsabilidad Social (RS) es definida de manera general en (Vallaeys, 2016) por el autor como:

...un nuevo sistema de gestión de la organización; una obligación universal para asegurar la sostenibilidad social y ambiental de nuestro modo de producción y consumo; un modo permanente de operar todas sus funciones basado en el diagnóstico y la buena gestión de sus impactos directos e indirectos.

La Responsabilidad Social ha sido adoptada por diversas empresas en las últimas décadas, a través de programas de extensión y acciones en beneficio social. Bajo el contexto empresarial, la RS es implementada por diversas razones, que van desde argumentos éticos, y la penetración de mercados hasta asegurar la lealtad de los clientes al proyectar una buena imagen. La percepción de la RS empresarial varía de país en país, en Reino Unido se le percibe como una herramienta de competitividad (Segal, 2004) y, en Francia se le percibe como una manipulación de la opinión pública (Wagenberg, 2006) y en América Latina como una buena conducta relacionada con obras caritativas (Correa, 2004). 
La problemática mundial actual ha llevado a repensar el papel de la universidad y a que ésta tome responsabilidad como agente de cambio que es. El entorno mundial orilla a la universidad a cambiar de un esquema tradicional de enseñanza clásico basado en transmisión de conocimientos técnicos (Jesús, Inés, Andrade, \& Martínez, n.d.) a un nuevo enfoque basado en competencias centradas en el alumno, cuyas dimensiones son: saber, saber hacer, saber ser y saber vivir y trabajar juntos (Romero Sánchez, Gleason Rodríguez, Rubio Barrios, \& Arriola Miranda, 2016); de modo que los egresados de las universidad no solo poseen conocimientos y habilidades técnicos de su campo, sino que son capaces de resolver un amplio abanico de situaciones de manera holística (Cabra, 2008). Por lo tanto, existe una estrecha relación entre las competencias de la persona y su disposición para actividades de RSU. Los autores de (Chumaceiro-Hernández, Herández, \& Chirinos-Noroño, 2018) exploran la importancia de la RSU orientada hacia la formación de estudiantes comprometidos con el medio ambiente, proponen la implementación de programas campañas para mejorar la rendición de cuentas de la universidad en este campo. La obtención de indicadores sobre RSU es una actividad importante (Aristimuño, Rodríguez, \& Guaita, 2011), por su parte, en (Baca-Neglia, Rondán-Cataluña, \& García-Del-Junco, 2017) los autores diseñan instrumentos y cuestionarios para medir la RSU en sus dimensiones: de gestión organizativa, gestión ambiental, docencia, investigación y de extensión; proponiendo los autores que otras universidades replique el trabajo realizado por ellos para validar sus resultados. Al ser la RSU una respuesta de la universidad, esta variará mucho de un lugar y tiempo a otros, por lo que es importante evaluar las diferentes experiencias (Casilla \& Camacho, 2012) y tratar de establecer la relación entre las diferentes concepciones de la RSU y sus evaluaciones, como en (Ramallo, 2015) proponen los autores. Puesto la RSU es realizada, en su mínima expresión, por la acción conjunta de individuos, es de interés el conocer las características de la población que es más propensa a realizar actividades de RSU, al respecto en (Rieckmann, 2012) los autores identificaron catorce principales competencias orientadas a la sostenibilidad. Sobre competencias y RSU, en (Alférez-Villarreal, 2014) el autor presenta una propuesta de actividades de extensión para diferentes asignaturas del Grado de Educación en la Universitat Internacional de Catalunya, comprobando si dicha implementación produce una mejora en la RSU y en ciertas competencias genéricas y profesionales.

En este trabajo se presenta una actividad de diagnóstico sobre las competencias genéricas de tres grupos de estudiantes de tres diferentes universidades, dos públicas y una privada, luego se presenta un análisis comparativo de los resultados obtenidos.

Las comunicaciones tendrán una extensión mínima de 5 folios y máxima de 15 ; y deberán constar de los siguientes apartados:

\section{Objetivos}

El objetivo general del este trabajo es implementar una metodología que permita obtener indicadores sobre el nivel de la RSU de alumnos y profesores en relación con sus competencias genéricas para tres diferentes universidades.

Como objetivos particulares se tienen los siguientes:

- Seleccionar y adaptar una encuesta que permita relacionar competencias genéricas con nivel de RSU.

- Seleccionar tres grupos de estudiantes de tres diferentes universidades.

- Aplicar la encuesta de manera digital.

- Realizar un análisis comparativo sobre los resultados de la encuesta. Desarrollo de la innovación 


\section{Desarrollo de la Innovación}

\section{Descripción y criterios}

En este estudio comparativo se propone la realización de una encuesta, que relaciona competencias genéricas con el nivel de RSU de estudiantes y docentes universitarios, de tres diferentes universidades. La encuesta fue tomada del trabajo realizado por (Alférez-Villarreal, 2014), y adaptada según recomendaciones de los autores de (Vallaeys et al., 2009). Las encuestas fueron aplicadas de manera digital empleando la plataforma de Microsoft Forms. Se seleccionó de manera digital para que el encuestado no se viera influenciado de ninguna manera por un encuestador, puesto que las preguntas abordan temas de comportamiento y percepción personales. En la Figura 1 se muestra los pasos seguidos para el desarrollo del presente trabajo.

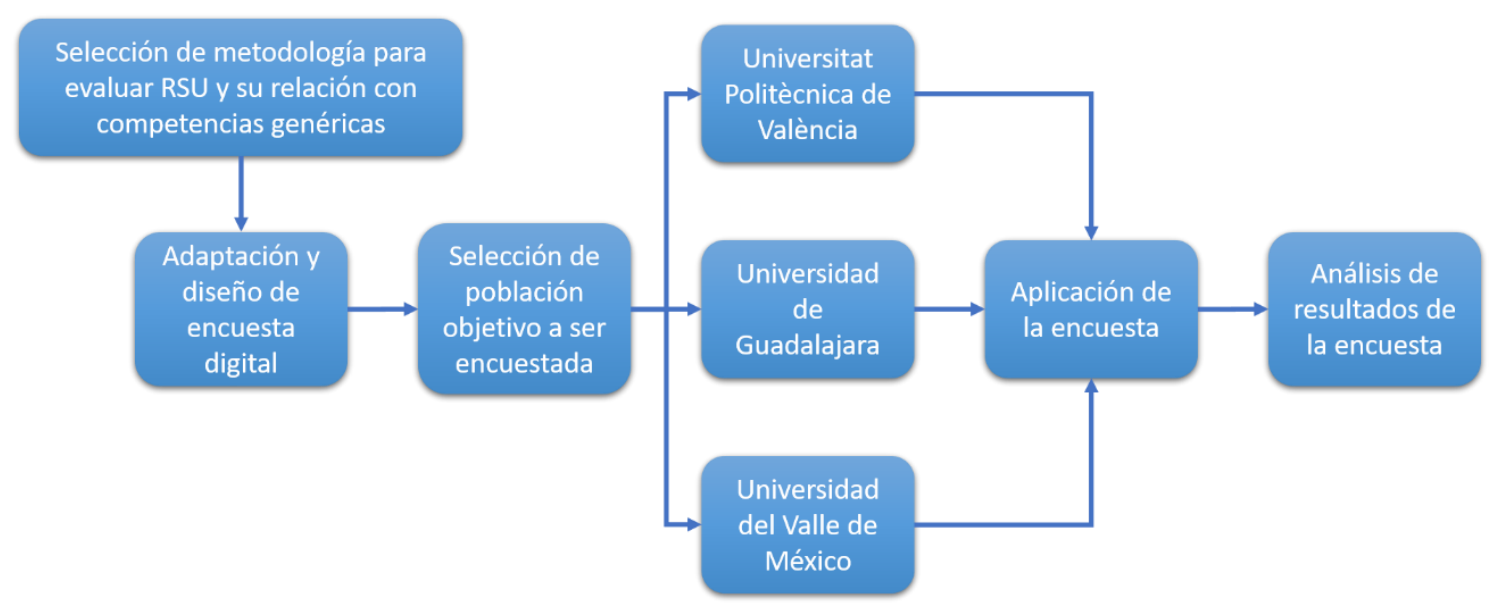

Fig. 1 Diagrama de proceso para el desarrollo del diagnóstico de las competencias relacionadas con la RSU para las universidades seleccionadas.

El cuestionario aplicado (el cual puede consultarse en la sección de Anexos) consta de 31 preguntas, las primeras 18 preguntas son demográficas y las restantes relacionan competencias genéricas y RSU.

\section{Relación entre las competencias genéricas y la RSU}

La definición de competencia está ligada al informe Delors, la Organisation for Economic Co-operation and Development (OECD) y el proyecto Tunning. En (Delors, 1996) se establece que las competencias tienen cuatro principales dimensiones: aprender a conocer, aprender a vivir juntos y aprender a ser; bajo esta perspectiva, la RSU tiene importancia desde el aprender a vivir juntos. Por su parte, la OECD en (Ananiadou \& Claro, 2009) se establece que las competencias son las acciones, modo de comportamiento y toma de decisiones ante diferentes escenarios y finalmente en el proyecto Tunning (González \& Wagenaar, 2003) para la educación en Europa se definen dos tipos de competencias: las profesionales y las genéricas. Las profesionales son aquellas que abonan al perfil técnico de egreso del estudiante, y las genéricas son las competencias que le ayudan a crecer como persona, a desenvolverse de una mejor manera ante otras personas, la sociedad y el medio ambiente con responsabilidad. El presente trabajo se centra por lo tanto en hacer una encuesta de diagnóstico por medio de preguntas clave que permitan conocer las competencias genéricas que ayudan a los estudiantes a comportarse con un mayor grado de RSU. Las preguntas fueron tomadas y modificadas del trabajo de (Alférez-Villarreal, 2014), son preguntas de escala del 1 al 6 , donde 1 es totalmente en desacuerdo y 6 es totalmente de acuerdo. La 
Tabla 1 muestra las preguntas relacionadas con las competencias genéricas seleccionadas y su relación con la RSU.

Tabla 1. Dimensiones de la competencias genéricas seleccionadas y su relación con la RSU para las preguntas de escala del cuestionario aplicado.

\begin{tabular}{lll}
\hline Dimensión de la competencia & Relación con la RSU & Preguntas de calificación con escala del 1 al 6
\end{tabular}

Saber (aspectos cognitivos de la Formación de la 1 . He tomado conciencia de la
$\begin{array}{lll}\text { competencia) } & \text { Responsabilidad Social } & \text { Responsabilidad Social que implica el }\end{array}$ hecho de ser universitario.

2. Soy consciente de que en la medida en que estudie y me prepare a fondo en la Universidad podré influir más en la mejora del conjunto de la sociedad.

3. Considero que la Responsabilidad Social es una competencia que se debe trabajar en la Universidad.

Saber hacer (aspectos Planteamiento del procedimentales de la competencia) ejercicio profesional desde el compromiso social

Saber ser (aspectos actitudinales de la competencia)

Saber vivir y trabajar juntos

Compromiso con los demás y con el entorno
Descubrimiento personal de los valores
4. Me planteo el ejercicio de mi profesión como una vocación de servicio y orientado al bien común.

5. Creo que mi realización personal y mi felicidad pasan por ser un profesional comprometido en la mejora del conjunto de la sociedad.

6. Creo que es realista afirmar que desde el ejercicio profesional es posible el compromiso social.

7. Reconozco la necesidad de abrirme a los otros, de ponerme en su lugar y buscar el bien común, por encima de intereses individualistas.

8. He aprendido a valorar más positivamente mi situación personal y el lugar que ocupo en el mundo, al abrirme a otras realidades distintas a las mías.

9. He experimentado en primera persona la felicidad que conlleva el servicio y la solidaridad.

10. Tengo una visión global de la situación actual del mundo y soy consciente de la necesidad urgente de un desarrollo sostenible.

11. Me cuestiono cuál es mi posicionamiento personal ante las injusticias sociales, ante el dolor ajeno.

12. Considero que una de mis obligaciones como persona es ayudar a los demás, desde el compromiso social.

Fuente: Elaboración propia adaptada de (Alférez-Villarreal, 2014). 


\section{Aplicación de la encuesta}

La encuesta se aplicó de manera digital empleando la plataforma Microsoft Forms, de tal manera que el encuestado no se viera influenciado de manera alguna por un entrevistador. La Figura 2 muestra el inicio del formulario.

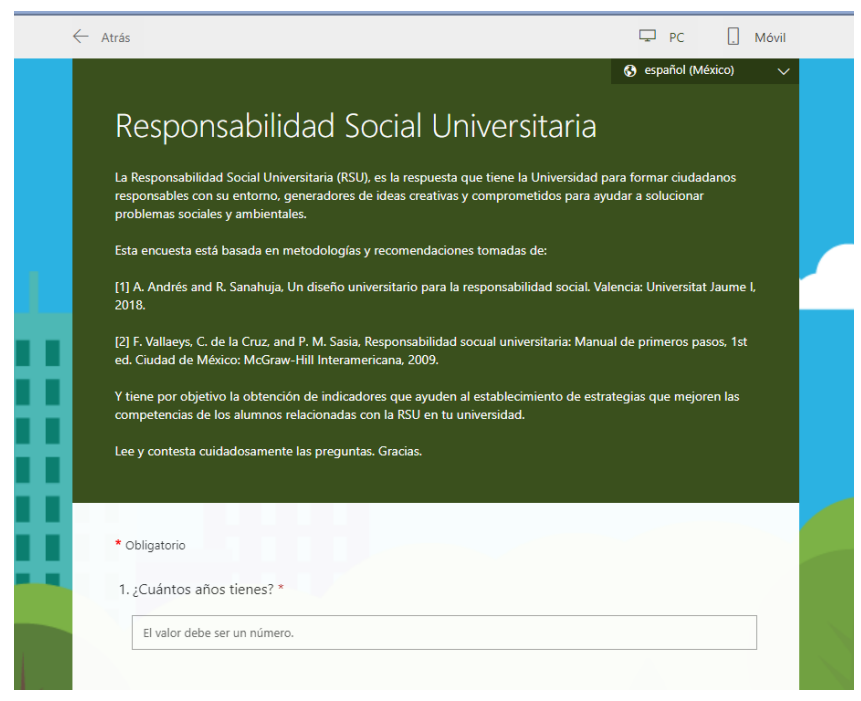

Fig. 2 Formulario online para el cuestionario de RSU, montado en Microsoft Forms.

Se seleccionaron tres universidades para aplicar el cuestionario.

- Universidad de Guadalajara (UdeG), universidad pública del Estado de Jalisco en México.

- Universitat Politècnica de València (UPV), universidad pública de la Comunidad Valenciana en España.

- Universidad del Valle de México (UVM), universidad privada del Estado de Jalisco en México.

Como se aprecia en la Tabla 2, donde se resume las principales características de la población encuestada, el número de encuestados fue mayor en la UPV, con una edad promedio de 25 años. Los encuestados de la UVM son los que más experiencia han tenido en voluntariados, mientras que lo que menos experiencia son los encuestados de la UdeG.

Tabla 2. Principales características de la población encuestada

\begin{tabular}{lccc}
\hline & $\begin{array}{c}\text { Universidad de } \\
\text { Guadalajara }\end{array}$ & $\begin{array}{c}\text { Universitat Politècnica } \\
\text { de València }\end{array}$ & $\begin{array}{c}\text { Universidad del Valle } \\
\text { de México }\end{array}$ \\
\hline Total, de encuestados & 24 & 37 & 25 \\
Edad promedio (años) & 23 & 25 & 19 \\
$\begin{array}{l}\text { Han realizado o } \\
\text { realizan algún } \\
\text { voluntariado }\end{array}$ & $29 \%$ & $38 \%$ & $72 \%$ \\
\hline
\end{tabular}




\section{Resultados y discusiones}

La primera parte del cuestionario de formulario online contiene preguntas de interés demográfico, pero indirectamente relacionadas con la RSU, como el desarrollo de competencias genéricas en la familia. La Figura 3 muestra el nivel de estudio máximo de los padres de los encuestados.

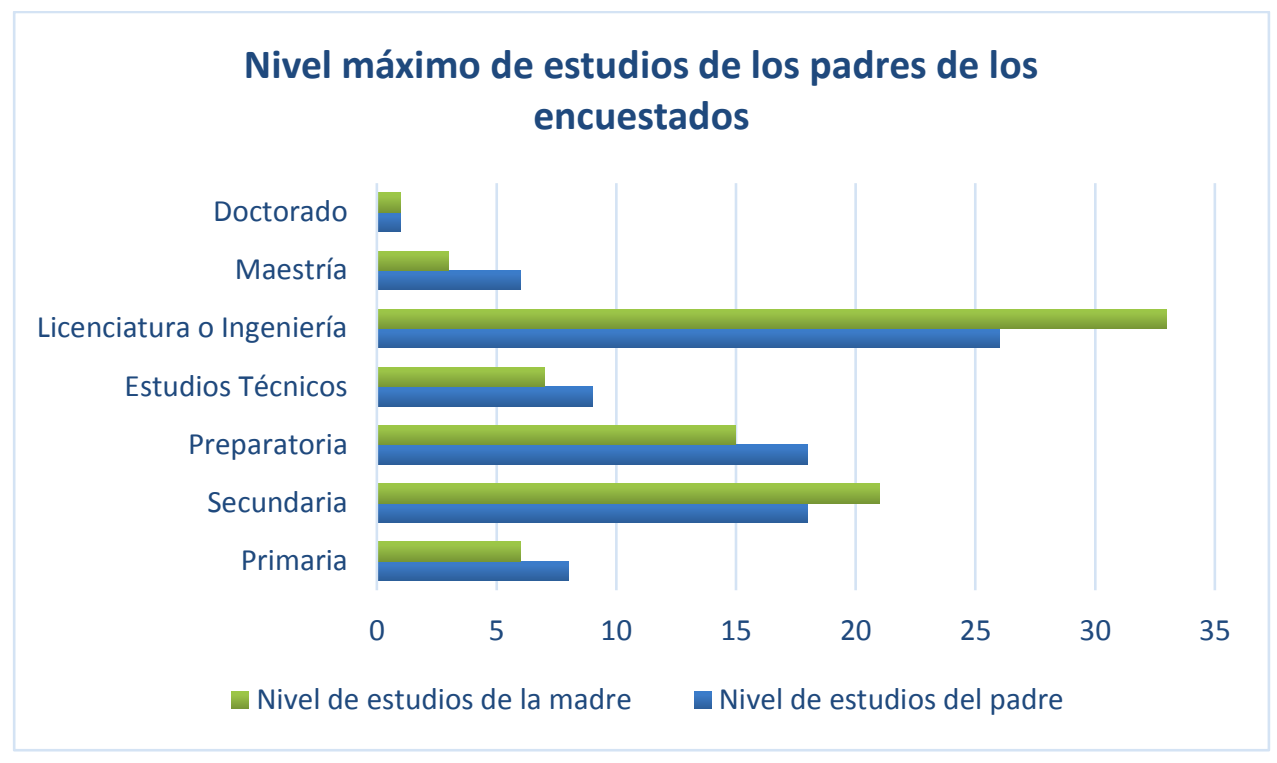

Fig. 3 Nivel de estudio máximo promedio de los padres de los encuestados.

Como puede apreciarse en la Figura 3, la mayoría de los padres de los encuestados obtuvieron un grado máximo de estudios de grado en licenciatura o ingeniería. Esto es un indicador de que al ser los padres los primeros formadores de las competencias genéricas en sus hijos, es lógico pensar que los padres les transmitirán además del conjunto de competencias genéricas familiares heredadas también aquellas que se adquieren o se refuerzan durante los estudios de grado en la universidad. Por lo que las estrategias de aprendizaje y reforzamiento de competencias genéricas durante los estudios de grado universitarios adquieren una mayor relevancia para la formación de las futuras generaciones.

Otra pregunta clave que se hizo al inicio del cuestionario fue si el encuestado había o no realizado alguna vez un voluntariado. La Figura 4 muestra la cantidad de personas que realizaron voluntariado para cada universidad.

Como se puede apreciar en la Figura 4, la UdeG y la UVM comparten porcentajes, pero contrarios. En la UdeG el $29 \%$ de los encuestados dijo haber realizado algún voluntariado, mientras que en la UVM el $72 \%$ lo ha hecho. Los mayores porcentajes de negación a voluntariados se encontraron en la UdeG y la UPV, con un $71 \%$ y $62 \%$ respectivamente, ambas universidades comparten el ser públicas, mientras que la UVM es una institución privada. 


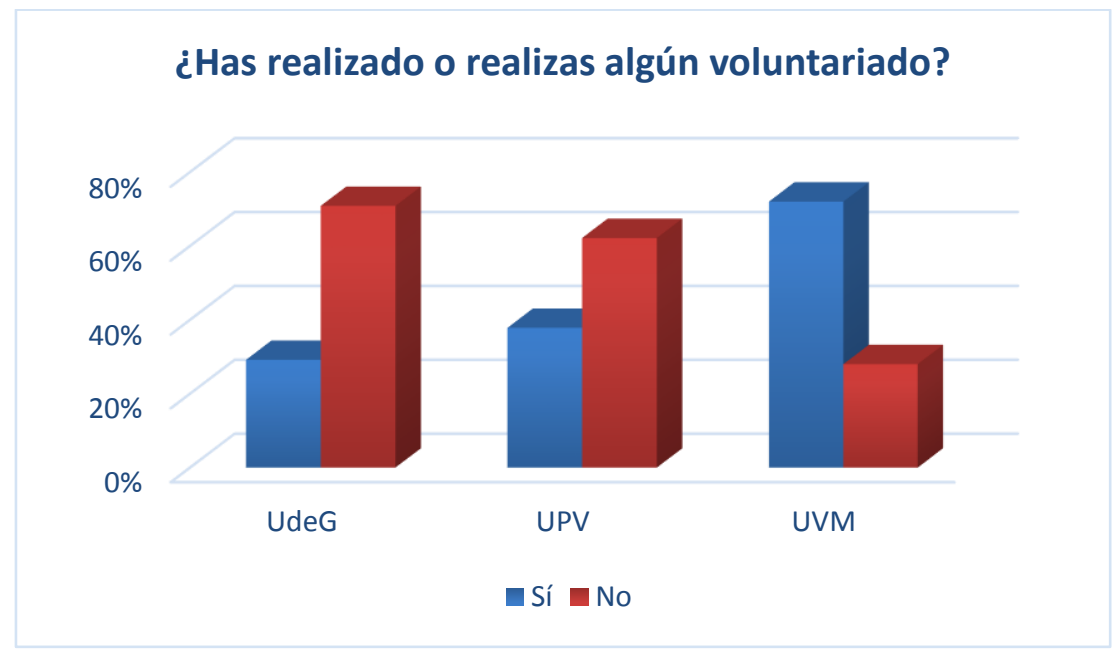

Fig. 4 Porcentaje de encuestados que alguna vez han realizado o realizan actualmente algún voluntariado.

\section{Compromiso con los demás y el entorno}

Este apartado resume los resultados para las tres universidades de las preguntas 10 a 12, mostradas con anterioridad en la Tabla 1, que están relacionadas con el nivel de compromiso de los encuestados para actuar con responsabilidad y respeto, individual y colectivamente. Como puede ver en la Figura 5, los encuestados de la UdeG y la UVM tienen una tendencia muy positiva al respecto, de entre el $30 \%$ y el 40\% para "Totalmente comprometido", mientras que los encuestados de la UPV son un poco más reservados, con menos del 10\% para "Totalmente comprometido".

UdeG

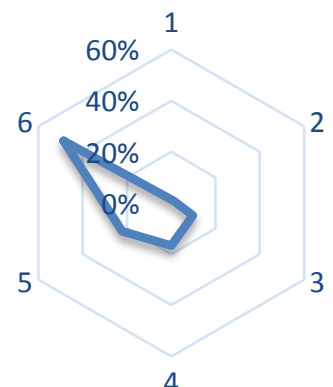

4

\section{UPV}

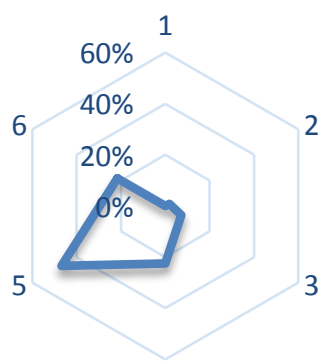

4

\section{UVM}

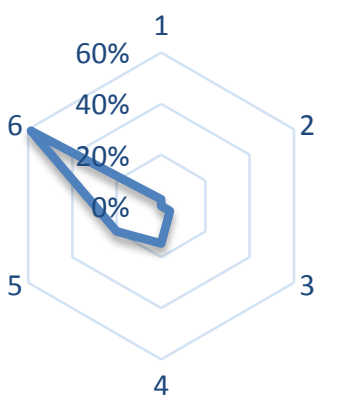

$1=$ Absolutamente no comprometido

2 = No comprometido

$3=$ Parcialmente no comprometido

$4=$ Parcialmente comprometido

5 = Comprometido

$6=$ Totalmente comprometido

Fig. 5 Comparativa del nivel de compromiso con los demás y el entorno en los encuestados de la UdeG, UPV y UVM.

\section{Descubrimiento personal y de los valores}

Las preguntas 7 a 9 de la Tabla 1 están relacionadas con la confianza en uno mismo, la expresión personal y la comunicación, así como el modo en que el encuestado identifica y clarifica valores. En la Figura 6 se muestran los resultados obtenidos del cuestionario online, se ve una clara tendencia similar entre la UdeG y la UVM, sin embargo, los encuestados de la UVM muestran tener una mayor disposición, del 40\% para 
"Totalmente dispuesto", en comparación la UPV donde solo el 10\% está "Totalmente dispuesto", y un 30\% para "Dispuesto". Esto puede ser un indicador de la predisposición cultural diferente entre México y España.

\section{UdeG}

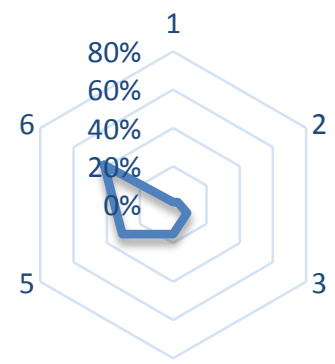

4
UPV

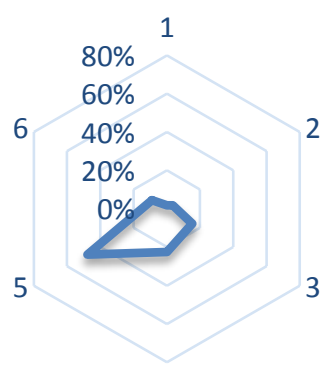

4
UVM

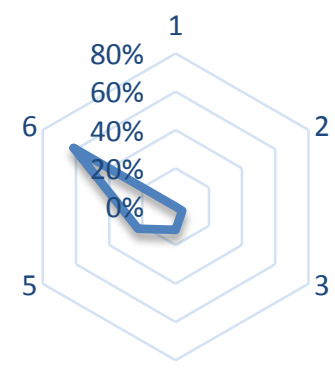

4

\footnotetext{
$1=$ Totalmente indispuesto

$2=$ Indispuesto

3 = Parcialmente indispuesto

$4=$ Parcialmente dispuesto

$5=$ Dispuesto

$6=$ Totalmente dispuesto
}

Fig. 6 Comparativa del nivel de disposición al descubrimiento personal y los valores en los encuestados de la UdeG, UPVy UVM.

\section{Formación de la Responsabilidad Social}

Las preguntas 1 a 3 de la Tabla 1 están relacionadas con el pensamiento crítico y sistémico, y la capacidad de resolver y gestionar problemas de manera holística en el encuestado. En la Figura 7 puede observarse cómo los encuestados de la UPV tienen una visión más conservadora, con alrededor del 10\% para "Totalmente de acuerdo" contra la cerca del 40\% para la UdeG y la UVM. respecto a la formación de la Responsabilidad Social.

\section{UdeG}

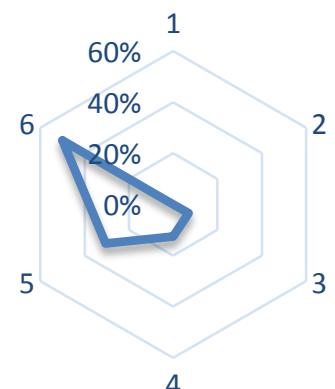

4

\section{UPV}

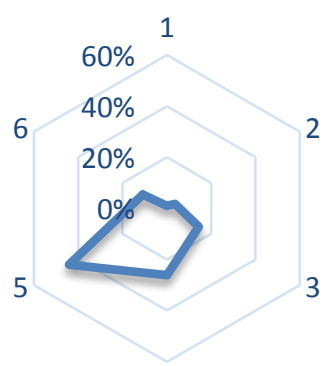

4

\section{UVM}

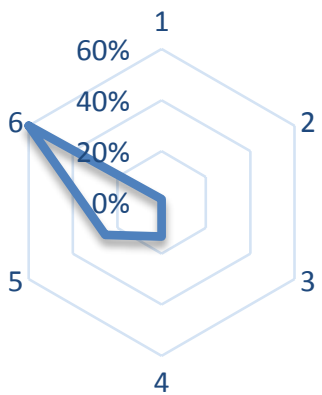

$1=$ Totalmente en desacuerdo

$2=$ En desacuerdo

$3=$ Parcialmente en desacuerdo

$4=$ Parcialmente de acuerdo

$5=$ De acuerdo

$6=$ Totalmente de acuerdo

Fig. 7 Comparativa de la Formación de la Responsabilidad Social en los encuestados de la UdeG, UPVy UVM. 


\section{Planteamiento del ejercicio profesional desde el compromiso social}

El hacer frente a situaciones de crisis es muy importante, sobre todo en casos de gran incertidumbre para tomar acciones con responsabilidad para uno mismo y con los demás, las preguntas X-X del cuestionario evalúan estos aspectos los encuestados, mostrándose los resultados en la Figura 8. Como puede apreciarse en la Figura 8, los encuestados de la UdeG tienen un enfoque equilibrado entre "De acuerdo" y "Totalmente de acuerdo" con el $20 \%$ aproximadamente para cada uno, mientras que en la UPV se observa una clara tendencia a "De acuerdo" con un $30 \%$ y en la UVM a "Totalmente de acuerdo" con cerca del $50 \%$.

\section{UdeG}

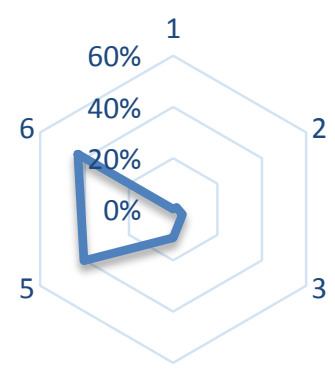

4
UPV

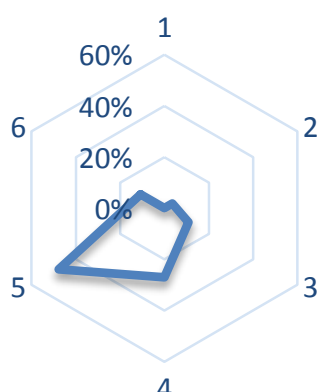

4
UVM

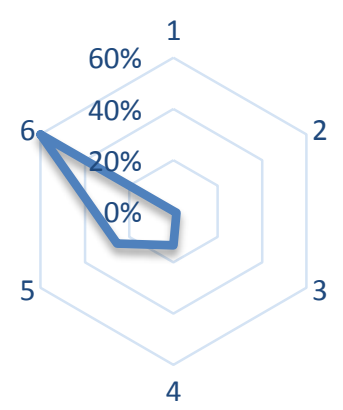

$1=$ Totalmente en desacuerdo

$2=$ En desacuerdo

$3=$ Parcialmente en desacuerdo

$4=$ Parcialmente de acuerdo

$5=$ De acuerdo

$6=$ Totalmente de acuerdo

Fig. 8 Comparativa del Planteamiento del ejercicio profesional desde el compromiso social para los encuestados, UdeG, UPV y $U V M$.

\section{Autopercepción del grado de Responsabilidad Social}

Por último, se preguntó al encuestado sobre la percepción personal de su persona y la Responsabilidad Social. En la Figura 9 se muestran los resultados de la autopercepción del grado de Responsabilidad Social para los encuestados de las tres universidades.

En la Figura 8 se aprecia que en la UdeG y la UPV se tienen auto percepciones similares del grado de Responsabilidad Social, con "Parcialmente de acuerdo" entre 35\% y 30\% respectivamente, y en "De acuerdo" con 30\% para las dos. Por su parte la UVM tiene un rango similar de cerca del $30 \%$ tanto para "Totalmente de acuerdo" y "De acuerdo". 
UdeG

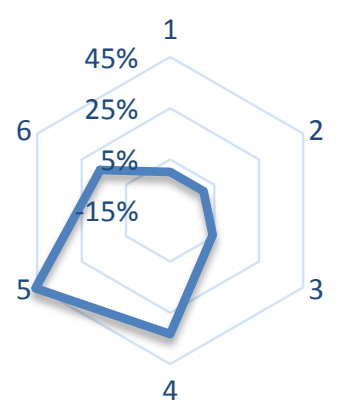

UPV

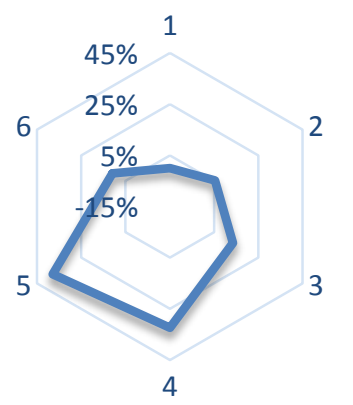

UVM

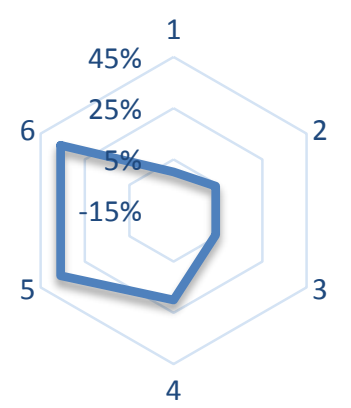

$1=$ Totalmente en desacuerdo

$2=$ En desacuerdo

$3=$ Parcialmente en desacuerdo

$4=$ Parcialmente de acuerdo

$5=$ De acuerdo

$6=$ Totalmente de acuerdo

Fig. 8 Comparativa del Planteamiento del ejercicio profesional desde el compromiso social para los encuestados de la UdeG, UPV y UVM.

\section{Conclusiones}

En el trabajo presentado se mostró una comparativa sobre los resultados de una encuesta que mide las competencias genéricas y su relación con las Responsabilidad Social Universitaria de tres diferentes universidades: dos mexicanas, una pública (UdeG) y una privada (UVM), y una universidad pública española (UPV). Se observó que los encuestados de las universidades UdeG y UVM tuvieron una tendencia a contestar más positivamente que los encuestados de la UPV, siendo esto en promedio más mesurados en cuanto a las respuestas. Por otro lado, los encuestados de la UdeG y la UPV tienen auto percepciones similares del grado de Responsabilidad Social, mostrando tendencias parecidas, en contraste con la UVM; siendo posiblemente la causa de esto que tanto la UPV como la UdeG son universidades públicas. El hecho de que, en las universidades públicas, UdeG y UPV, la percepción de Responsabilidad Social sea similar y menos optimista que en la universidad privada, UVM, puede deberse a que la UVM al ser una universidad privada trabajo más a un modo de empresa, donde es importante cuidar la imagen pública respecto a la Responsabilidad Social, trasladando la dimensión de Responsabilidad Social Empresarial a Responsabilidad Social Universitaria de manera más efectiva que las universidades públicas. Los resultados del presente trabajo sugieren que tanto en la UPV, como en la UdeG en mayor medida, es importante establecer estrategias que ayuden a reforzar actividades genéricas que coadyuven a los estudiantes a un mayor involucramiento en la Responsabilidad Social Universitaria. 


\section{Referencias}

Alférez-Villarreal, A. (2014). Responsabilidad Social Universitaria en la formación curricular y la participación social: El caso de la Facultad de Educación de la Universitat Internacional de Catalunya Azul Alférez Villarreal Dipòsit Legal : B 24465-2014. Universitat Internacional de Cataluya.

Ananiadou, K., \& Claro, M. (2009). 21st century skills and competences for new millennium learners in OECD countries.

Aristimuño, M., Rodríguez, C., \& Guaita, W. (2011). La Responsabilidad Social Universitaria : Indicadores para su evaluación en Instituciones de Educación Superior. Ninth LACCEI Latin American and Caribbean Conference, Engineering for a Smart Planet, Innovation, Information Technology and Computactional Tools for Sustainable Development, (Agosto 3-5), 1-7. Retrieved from http://www.laccei.org/LACCEI2011Medellin/published/UM210_Aristimuno.pdf

Baca-Neglia, H. Z., Rondán-Cataluña, F. J., \& García-Del-Junco, J. (2017). Propuesta de medición de la responsabilidad social universitaria. Espacios, 38(43).

Cabra, F. (2008). La evaluación y el enfoque de competencias. Revista Escuela de Administración de Negocios, Universidad EAN Colombia, (0120-8160), 91-105.

Casilla, D., \& Camacho, H. (2012). Evaluación de la responsabilidad social universitaria. Opcion, 28(69), $452-465$.

Chumaceiro-Hernández, A., Herández, J. J., \& Chirinos-Noroño, E. (2018). Responsabilidad social universitaria, desarrollo sostenible y ciudadanía ambiental. Cuadernos Del RSO, 4(June), 53-64.

Correa, M. E. (2004). Responsabilidad social corporativa en América Latina: una visión empresarial (Vol. 85). United Nations Publications.

Delors, J. (1996). Informe a la UNESCO de la Comisión Internacional sobre la educación para el siglo XXI, presidida por JACQUES DELORS La Educación Encierra un Tesoro. España: Santillana Ediciones UNESCO.

González, J., \& Wagenaar, R. (2003). Tuning educational structures in Europe. University of Deusto Bilbao.

Jesús, D., Inés, M., Andrade, R., \& Martínez, R. (n.d.). Didáctica: Docencia y método. Una visión comparada entre la universidad tradicional y la multiversidad compleja. Retrieved from http://www.ula.ve

Leal, J., \& Mira, M. (2016). L'insegnamento superiore nella storia della Chiesa: scuole, maestri e metodi. EDUSC. Retrieved from https://books.google.com.mx/books?id=0lclMQAACAAJ

Ramallo, M. (2015). La evaluación de la Responsabilidad Social Universitaria. Debate Universitario, 7(noviembre), $2-7$.

Rieckmann, M. (2012). Future-oriented higher education: Which key competencies should be fostered through university teaching and learning? Futures, 44(2), 127-135. https://doi.org/10.1016/j.futures.2011.09.005

Romero Sánchez, M. D. C., Gleason Rodríguez, M., Rubio Barrios, J. E., \& Arriola Miranda, M. A. (2016). VALIDACIÓN DE UN MODELO DE COMPETENCIAS DOCENTES EN UNA UNIVERSIDAD PRIVADA MEXICANA. Revista Digital de Investigación En Docencia Universitaria, (1), 1. https://doi.org/10.19083/ridu.10.455

Segal, J.-P. (2004). Pluralité des lectures politiques de la responsabilité sociale de l'entreprise en Europe. Documento de Trabajo.

UNAM. (2020). Cédula Real sobre la fundación de la Universidad de México | Portal UNAM. Retrieved March 11, 2020, from https://www.unam.mx/acerca-de-la-unam/unam-en-el-tiempo/cronologia-historica-de-launam/cedula-real-sobre-la-fundacion

Universidad de Salamanca. (2020). Conoce más información sobre Salamanca y la Universidad de Salamanca | Salamanca-University.org. Retrieved March 11, 2020, from https://www.salamancauniversity.org/universidad-salamanca.htm

Vallaeys, F. (2016). Introducción a la Responsabilidad Social Universitaria. Barranquilla-Cúcuta: Ediciones Universidad Simón Bolívar. 
Vallaeys, F., de la Cruz, C., \& Sasia, P. M. (2009). Responsabilidad socual universitaria: Manual de primeros pasos (1st ed.). Ciudad de México: McGraw-Hill Interamericana.

Wagenberg, A. (2006). La narrativa de la responsabilidad social empresarial. Cuadernos Latinoamericanos de Administración, 2(2), 113-120.

\section{Anexos}

\section{Cuestionario aplicado}

1) ¿Cuántos años tienes?

2) Sexo:
a. Femenino

b. Masculino

3) ¿Qué carrera cursas o cursaste?

4) ¿Cuál es tu universidad?

a. Universidad de Guadalajara

b. Universidad del Valle de México

c. Universitat Politècnica de València

5) ¿Cuál es tu Centro Universitario / Campus?

6) ¿En qué municipio vives?
a. Guadalajara
b. Zapopan
c. San Pedro Tlaquepaque
d. Tonalá
e. Tlajomulco de Zúñiga
f. El Salto
g. Ixtlahuacán de los Membrillos
h. Juanacatlán
i. Otro fuera del Área Metropolitana de Guadalajara, o fuera de México.

7) ¿Eres...?
a. Estudiante
b. Becario
c. Profesor
d. Personal contratado en la universidad
e. En prácticas en la universidad

8) ¿Tienes alguna asignatura pendiente en tus estudios?
a. Ninguna
b. De 1 a 4
c. Más de 4

9) Indica el nivel de estudios de tu padre:
a. Educación primaria
b. Educación secundaria
c. Educación preparatoria
d. Estudios técnicos
e. Licenciatura o ingeniería
f. Maestría
g. Doctorado

10) Indica el nivel de estudios de tu madre:
a. Educación primaria
b. Educación secundaria
c. Educación preparatoria
d. Estudios técnicos
e. Licenciatura o ingeniería
f. Maestría
g. Doctorado 
11) Actualmente ¿Tu padre trabaja?
a. Sí
b. No

12) ¿Tu madre trabaja?
a. Sí
b. No

13) ¿Y tú, trabajas?
a. Sí
b. No

14) ¿Consideras que tu familia te ha comunicado valores altruistas?
a. Sí
b. No

15) ¿Te consideras una personas motivada, motivadora y participativa?
a. Sí
b. No

16) ¿Te consideras una persona empática?
a. Sí
b. No

17) ¿Participas de la cultura del cambio social, es decir, consideras que las cosas pueden ser de una distinta manera, porque está en nuestras manos cambiarlas?
a. Sí
b. No

18) ¿Has realizado o realizas algún voluntariado?
a. Sí
b. No

\section{Sección de preguntas de escala del 1(totalmente en desacuerdo) al 6 (totalmente de acuerdo)}

19) Considero urgente el tener una visión global del mundo y un desarrollo sostenible.

20) Frecuentemente cuestiono mi posicionamiento personal ante las injusticias sociales, ante el dolor ajeno.

21) Considero que una de mis obligaciones como persona es ayudar a los demás, desde el compromiso social.

22) Habitualmente reconozco la necesidad de abrirme a los otros, de ponerme en su lugar y buscar el bien común, por encima de intereses individualistas.

23) Valoro mi situación personal y el lugar que ocupo en el mundo, al abrirme a otras realidades distintas a las mías.

24) He experimentado en primera persona la felicidad que conlleva el servicio y la solidaridad.

25) Soy consciente de la Responsabilidad Social que implica el hecho de ser universitario.

26) En la medida en que estudie y me prepare a fondo en la Universidad podré influir más en la mejora del conjunto de la sociedad.

27) La Responsabilidad Social es una competencia que se debe trabajar en la Universidad.

28) El ejercicio de la profesión es una vocación de servicio orientado al bien común.

29) La realización personal y felicidad están relacionadas con el nivel de compromiso en la mejora del conjunto de la sociedad. 
30) Desde el ejercicio profesional es posible el compromiso social.

31) Tengo un alto grado de Responsabilidad Social como universitario, en estos momentos. 